Chapter 4

\title{
Compression Neuropathies
}

\author{
Javier López Mendoza and \\ Alexandro Aguilera Salgado \\ Additional information is available at the end of the chapter \\ http://dx.doi.org/10.5772/55316
}

\section{Introduction}

Upper extremity compression neuropathies are among the most common disorders in plastic surgery, especially in patients with predisposing occupations or with certain medical disorders. In the past two decades a notable increase in the incidence of this entity has occurred. Therefore, it is mandatory to achieve a prompt diagnosis because they can produce important motor and sensory deficiencies that need to be treated before the development of complications, since, despite the capacity for regeneration bestowed on the peripheral nervous system, functions lost as a result of denervation are never fully restored.

\section{Etiology}

There are many different situations that may be a direct cause of nerve compression. Anatomically, nerves can be compressed when traversing fibro-osseous tunnels, passing between muscle layers, through traction as they cross joints or buckling during certain movements of the wrist and elbow. Other causes include trauma, direct pressure and space-occupying lesions at any level in the upper extremity.

There are other situations that are not a direct cause of nerve compression, but may increase the risk and may predispose the nerve to be compressed specially when the soft tissues are swollen like synovitis, pregnancy, hypothyroidism, diabetes or alcoholism [1].

Physicians in touch with patients who suffer from upper extremity compression neuropathies must apply all of their skills to correctly distinguish symptomatic nerve entrapment form other neurologic entities such as myelopathy, braquial plexopathy, radiculopathy, and other central nervous system disorders, that can mimic peripheral nerve entrapment. 
Besides these pathologies, the differential diagnosis must include painful rheumatologic and orthopedic disorders; and other psychological entities, such as somatoform and factitious disorders.

\section{Pathophysiology}

When nerve fibers undergo compression, the response depends on the force applied at the site and the duration. Acute, brief compression results in a focal conduction block as a result of local ischemia, being reversible if the duration of compression is transient. On the other hand, if the focal compression is prolonged, ischemic changes appear, followed by endoneurial edema and secondary perineurial thickening. These histological alterations will aggravate the changes in the microneural circulation and will increase the sensitivity of the neuron sheath to ischemia. If the compression continues, we will find focal demyelination, which typically results in a greater involvement of motor than sensory nerve fibers. Even at this point clinical and electrophysiologic signs can resolve within a period of weeks to months.

As the duration of compression increases beyond several hours, more diffuse demyelination will appear, being the last event an injury to the axons themselves. This process begins at the distal end of compression or injury, a process termed wallerian degeneration. These neural changes may not appear at a uniform fashion among the whole neural sheath depending on the distribution of the compressive forces, causing mixed demyelinating and axonal injury resulting from a combination of mechanical distortion of the nerve, ischemic injury, and impaired axonal flow [2].

\section{Clinical evaluation}

\subsection{History}

We must begin with a complete history of the patient, asking about preexisting diseases that may be a direct cause of the neuropathy or may exacerbate it like diabetes, hypothyroidism, alcoholism, rheumatologic or orthopedic problems and any history of trauma or surgeries that may explain his or her symptoms.

The symptoms we may find in our patients will depend on diverse factors, mainly in the nature of the nerve involved, if it is primary motor, sensitive or both, and the anatomic location of the site of compression. The principal affections we will find will be hypoesthesia of the territory of the sensitive nerve or a complete anesthesia in more chronic conditions. In case of motor nerve compression, symptoms will be related to a progressive loss of function to a complete muscular atrophy in severe cases.

We need to investigate when did the symptoms began, if they were progressive or sudden, which movements are limited or impaired, if hypoesthesia or complete loss of sensation is 
present, accompanying symptoms, and if they have improved with time or with a particular action taken by the patient.

\subsection{Physical exam}

Besides a complete history of our patient, a thorough nerve exam needs to be addressed based on the knowledge of the upper extremity nerves anatomy in order to determine the possible site of compression. Our physical exam must include a sensitive and a motor evaluation of the complete upper extremity, beginning with the evaluation of specific movements of the shoulder, arm, forearm, elbow, wrist and digits to determine which muscles are affected and the range of motion of each of these muscles. Next, the sensitivity must be tested, light touch, pain, pressure, vibration and two-point discrimination among the specific distribution of the main nerve involved. There are some complementary tests we may apply in order to guide our exam according to the nerve we think is involved. These specific tests will be discussed further in the chapter [3].

\section{Electrophysiology}

Electrophysiologic testing is part of the evaluation, but it never substitutes a complete history and a thorough physical examination. These tests can detect physiologic abnormalities in the course of motor and sensory axons. There are two main electrophysiologic tests: needle electromyography and nerve conduction, which permit differentiating between a focal mononeuropathy, a radiculopathy, and a plexopathy, or the discovery of a more diffuse process, such as a systemic peripheral neuropathy or motor neuron disorder.

The electromyography detects the voluntary or spontaneous generated electrical activity. The registry of this activity is made through the needle insertion, at rest and during muscular activity to assess duration, amplitude, configuration and recruitment after injury. Recruitment will be affected if demyelination occurs, but will not result in abnormal spontaneous activity. Meanwhile, axonal injury will result in both recruitment and abnormal spontaneous activity, which will not be seen on needle electromyography until 2 weeks after the initial insult [4].

Nerve conduction assesses for both sensory and motor nerves. This study consists in applying a voltage simulator to the skin over different points of the nerve in order to record the muscular action potential, analyzing the amplitude, duration, area, latency and conduction velocity. The amplitude indicates the number of available nerve fibers. Some authors consider diminished amplitude below $50 \%$ to be suggestive of compression. In such cases, we will find a normal response to distal stimulation but no response proximal to the site of entrapment. If the compression progresses, our results will be compatible with axonal degeneration with diminished amplitude of the response with relative preservation of the conduction velocity and distal latency until the remaining axons are completely damaged [5]. 


\section{Median nerve}

\subsection{Anatomy}

The median nerve is formed in the axilla by a branch each from the medial and lateral chords of the brachial plexus, receiving fibers from C6, C7, C8 and T1 roots. It arises anterior to the axillary artery, descending distally through the arm lateral to the brachial artery till it reaches the medial aspect of the arm. It is important to know that the median nerve has no branches above the cubital fossa. It enters the cubital fossa lateral to the brachialis tendon passing between the two heads of the pronator teres giving off the anterior interosseus branch (Figure 1).

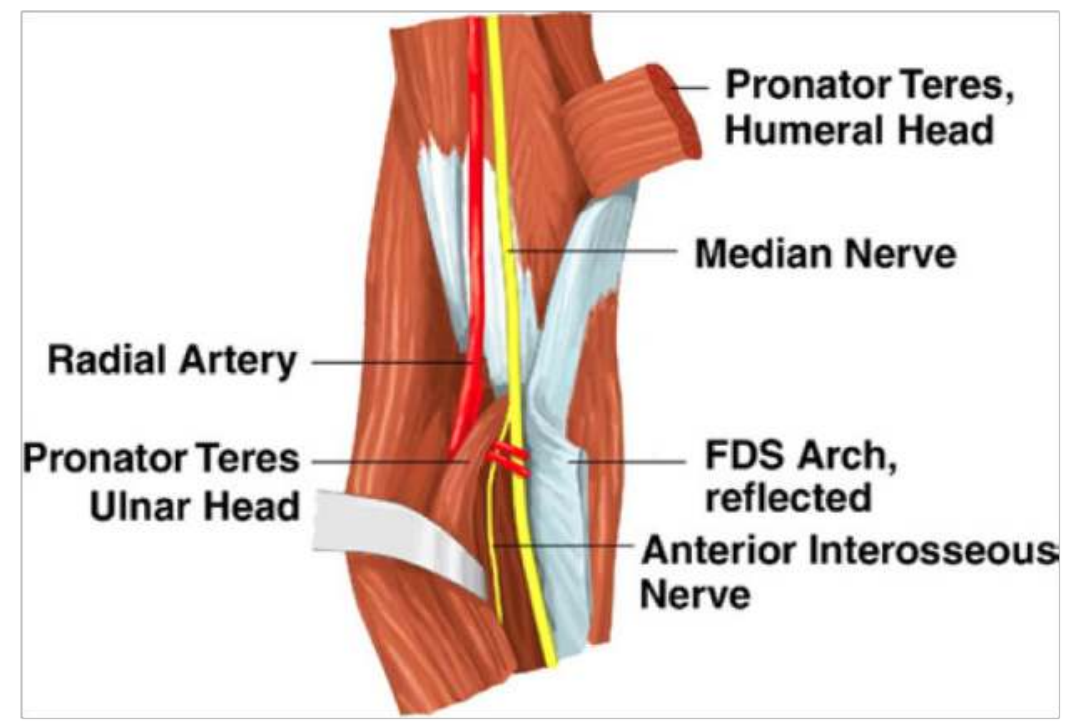

Figure 1. Median nerve descending lateral to brachial artery, giving off the anterior interosseus branch between the two heads of the pronator teres.

The nerve continues in the forearm between the flexor digitorium profundus and flexor digitorium superficialis, giving off above the wrist the palmar cutaneous branch that supplies the skin of the central portion of the palm. In the forearm it supplies the pronator teres, flexor carpi radialis, flexor digitorium superficialis and profundus, flexor pollicis longus and pronator quadratus [6] (Figure 2). 


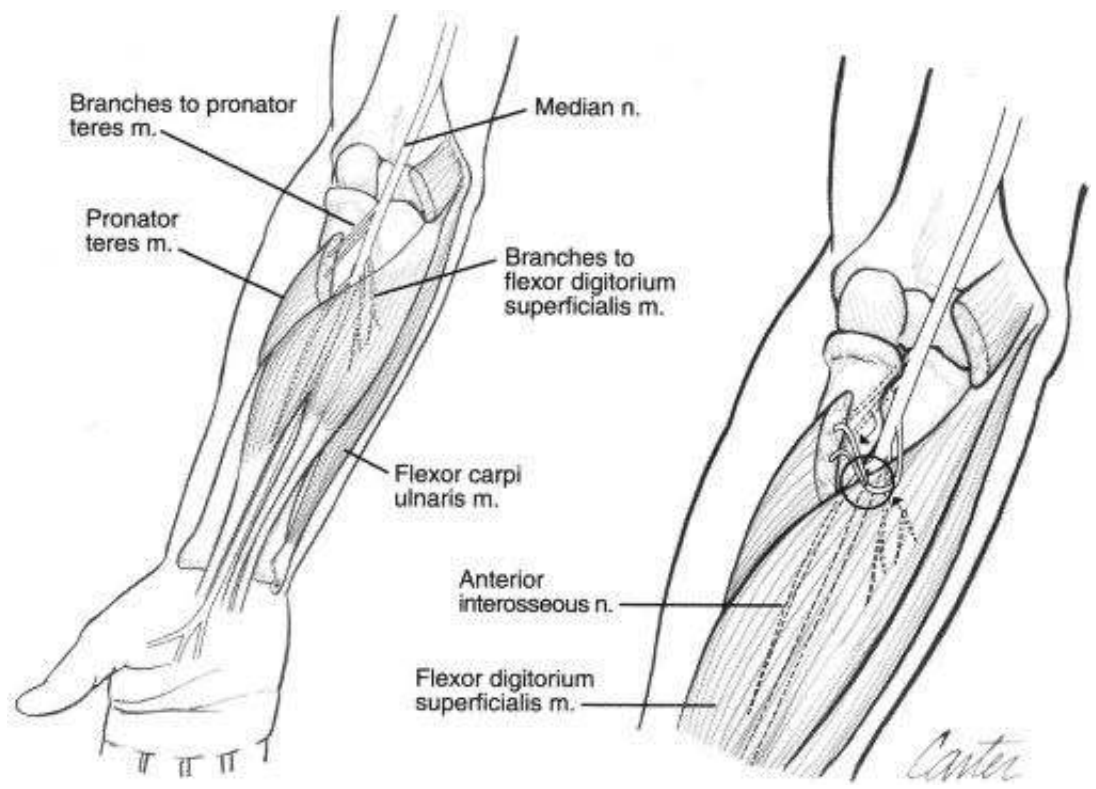

Figure 2. Branches and supplied muscles by the median nerve in the forearm.

Finally, it enters the hand through the carpal tunnel, running beneath the transverse carpal ligament, superficial to nine tendons: four of the flexor digitorium superficialis, four of the flexor digitorium profundus and one of the flexor pollicis longus. Distally it supplies the thenar eminence muscles and the lateral two lumbricalis, providing sensation to the first 3 digits and the lateral aspect of the fourth digit. (Figure 3).

\subsection{Median nerve entrapment}

There are three well-described entrapment syndromes involving the median nerve or its branches, namely pronator teres syndrome, anterior interosseous syndrome and carpal tunnel syndrome according to the level of entrapment. Each one of these syndromes presents with different clinical signs and symptoms, electrophysiologic results and requires different techniques for their release.

\subsection{Pronator teres syndrome (Proximal compression)}

\subsubsection{Sites of compression}

This is the most proximal compression site of the median nerve. It is due to compression of the median nerve as it passes through pronator teres. It may also be compressed upon the lacertus fibrosus (fascial sheet attached to biceps tendon), at the arched origin of the flexor digitorium superficialis or at the ligament of Struthers (connects medial epicondyle with a supracondylar process of humerus). 


\subsubsection{Diagnosis}

The onset is insidious and is suggested when the early sensory disturbances are greater on the thumb and index finger, mainly tingling, numbness and dysaesthesia in the median nerve distribution. Patients will also complain of increased pain in the proximal forearm and greater hand numbness with sustained power gripping or rotation because these movements tighten the fibrous origin of the superficial flexor muscles beneath which the median nerve passes. There is no nocturnal preference.

In the physical exam we may find a positive Tinel's sign at site of proximal compression within the antecubital fossa, negative over the carpal tunnel. Phalen's test will be negative. Generally, the neurophysiological exam will be normal, although in severe cases we may find fibrillations and sharp positive spikes in pronator quadratus and flexor pollicis longus.

\subsubsection{Treatment}

Surgical decompression is the definitive treatment. The incision should be distal to the elbow, oblique and parallel to the proximal margin of the pronator teres muscle, followed by an external neurolysis of the nerve performed proximally between the two heads of the pronator teres, and distally as it passes beneath the flexor digitorium superficialis muscle.

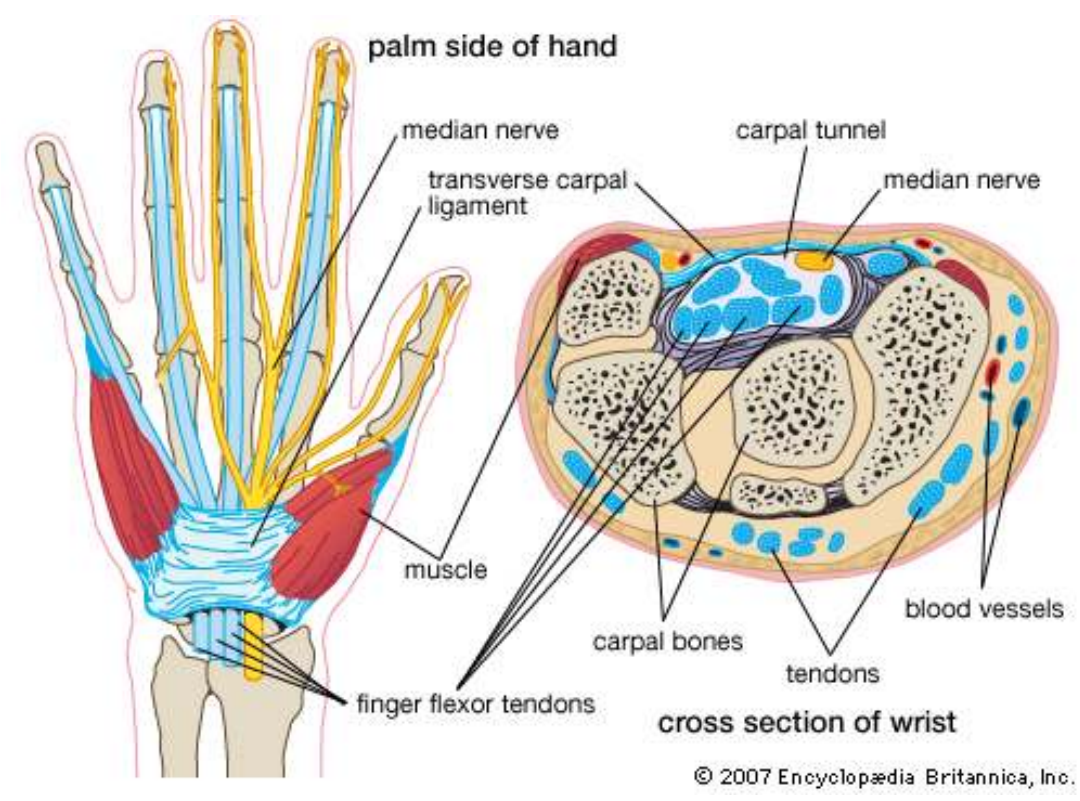

Figure 3. Carpal tunnel limits and branches of the median nerve in the hand. 


\subsection{Anterior interosseous syndrome}

\subsubsection{Innervation}

The anterior interosseous nerve classically innervates these muscles: flexor pollicis longus, pronator quadratus and the radial half of flexor digitorium profundus. These muscles are in the deep level of the anterior compartment of the forearm.

\subsubsection{Causes of compression}

The most common cause of this syndrome is a spontaneous fracture, probably due to brachial neuritis. Other causes include a space-occupying lesion, open fractures, elbow dislocation, compartment syndrome affecting the flexor compartment of the forearm, compression by the deep head of the pronator teres, the arch of flexor digitorium superficialis, or by Gantzer's muscle (accessory head of flexor pollicis longus).

\subsubsection{Diagnosis}

It presents principally as weakness of the index finger and thumb, and the patient may complain of diffuse pain in the proximal forearm, which may be exacerbated during exercise and diminished with rest. The vast majority of patients begin with pain in the upper arm, elbow and forearm, often preceding the motor symptoms. Pain is a common feature of anterior interosseus nerve compression, but it is not a predictive sign for differentiating an inflammatory from a mechanical origin.

During physical exam, the patient will be unable to bend the tip of the thumb and tip of index finger. The typical symptom is the inability to form an " $\mathrm{O}$ " with the thumb and index finger. Since flexor pollicis longus and flexor digitorium profundus to the index and middle finger are paralyzed, the patient will not be able to flex the interphalangeal joint of the thumb and the distal interphalangeal joint of the index finger. Sometimes, the motor branches to pronator teres, flexor carpi radialis and/or palmaris longus are also involved. [7] Spinner [8] has described a sign in which upon making a fist, the tips of the index finger and thumb remain conspicuously excluded. The examination of the pronator quadratus is difficult and unreliable. With the elbow bent at $90^{\circ}$, the patient is asked to forcibly pronate the forearm against resistance of the examiner.

The anterior interosseus nerve provides no sensory fibers to the skin, therefore, the sensation and sweating in the median nerve distribution is preserved. Abnormal sensibility in the median nerve distribution in the presence of an anterior interosseus syndrome, suggests a proximal median compression neuropathy involving fascicles of the anterior interosseus nerve. [9]

Electrophysiologic tests may reveal denervation and weakness of the muscles innervated by the anterior interosseous nerve. Other studies like an MRI should be necessary in order to discard space-occupying lesions and the involvement of bone and other structures. 


\subsubsection{Treatment}

If the onset was spontaneous and there is no evident lesion on MRI, supportive care and corticosteroid injections with observation for 4 to 6 weeks is usually accepted management. The degree of recovery is unpredictable. If the symptoms continue we may continue with a surgical treatment where a detachment or resection of the deep head of the pronator teres muscle is performed. If there is no evident recovery, we may have to consider tendon transfers.

\subsection{Carpal tunnel syndrome}

This is the most frequently encountered compression neuropathy in the upper limb. It is a mechanical compression of the median nerve through the fixed space of the rigid carpal tunnel. The incidence in the United States has been estimated at 1 to 3 cases per 1,000 subjects per year, with a prevalence of 50 cases per 1,000 subjects per year. [10] It is more common in women than in men (2:1), perhaps because the carpal tunnel itself may be smaller in women than in men. The dominant hand is usually affected first and produces the most severe pain. It usually occurs in adults, being the peak age range for development 45 to 60 years, and only $10 \%$ of patients are younger than 30 years. The risk of developing carpal tunnel syndrome is not confined to people in a single industry or job, but it is especially common in those performing assembly line work, manufacturing, sewing, cleaning and poultry or fish packing.

\subsubsection{Anatomy}

The carpal tunnel runs beneath the transverse carpal ligament, which transversely connects the pisiform, hamate, scaphoid and trapezium and longitudinally connects the deep fascia of the forearm and the palmar fascia. It contains the median nerve, 9 tendons previously described and the motor branch of the median nerve. There are three major patterns of branching of the recurrent motor branch: extraligamentous (50\%), subligamentous (31\%) and transligamentous (23\%) [11].

\subsubsection{Carpal tunnel pressure}

The lowest carpal tunnel pressure at rest with wrist in neutral position is $2.5 \mathrm{mmHg}$. In full wrist flexion it normally rises up to $30 \mathrm{mmHg}$. In patients with carpal tunnel syndrome, this pressure rises to $30 \mathrm{mmHg}$ and $90 \mathrm{mmHg}$ respectively (Phalen's test provokes this rise in pressure).

\subsubsection{Etiology}

There is still some controversy among the activities that may be a direct cause of carpal tunnel syndrome. It is believed to be idiopathic in the majority of cases and it has been related to repetitive prolonged wrist extension causing mechanical irritation, synovitis and eventually compressive neuropathy of the median nerve. Trauma can be another cause of this syndrome mainly among $5 \%$ of wrist fractures and $60 \%$ of lunate dislocations. Other rare disorders include renal failure and haemodialysis, hypothyroidism, pregnancy and some spaceoccupying lesions like ganglions and nerve tumours. 


\subsection{Anomalous interconnections}

In some cases we may find these anomalous interconnections that may explain some clinical findings not attributable to the median nerve like little finger numbness in carpal tunnel syndrome:

- Martin Gruber: Motor interconnections from median to ulnar nerve in forearm.

- Richie-Cannieu: Motor and sensory interconnections from median to ulnar nerve in the hand.

\subsubsection{Diagnosis}

It is mainly clinic, but complementary electrophysiologic tests should be ordered. It is typically first manifested by numbness, discomfort and parestesias of the thumb, index finger, middle finger and the radial side of the ring finger. As the symptoms progress, the patient may be awakened from sleep, referring constant numbness and pain. Pain may develop on the anterior wrist or at distal forearm at the carpal tunnel entrance (Durkin sign) and may be aggravated by elevation of the hand. Skin sensibility is not disturbed in the distribution of the palmar cutaneous branch as this branch is subcutaneous and does not pass through the carpal tunnel. Phalen and Tinel tests are highly reliable for diagnosis of carpal tunnel syndrome. If both tests are positive, there is a $91 \%$ chance of an accurate diagnosis. In advanced stages of carpal tunnel syndrome we may find thenar atrophy, which is associated with axonal damage [12].

Graham et al, developed a list of 6 clinical criteria (CTS-6) for the diagnosis of carpal tunnel syndrome, having all of them a statistically significant probability of being associated with this entity [13] (Table 1).

Electrodiagnostic studies are reliable for evaluation of suspected carpal tunnel syndrome, but in questionable cases, clinical evaluation supersedes these studies. Abnormalities on electrophysiologic testing, in association with specific symptoms and signs, are considered the criterion standard for carpal tunnel syndrome diagnosis. Electrophysiologic testing also can provide an accurate assessment of how severe the damage to the nerve is, thereby directing management and providing objective criteria for the determination of prognosis. Carpal tunnel syndrome is usually divided into mild, moderate and severe. In general, patients with mild carpal tunnel syndrome have sensory abnormalities alone on electrophysiologic testing, and patients with sensory plus motor abnormalities have moderate carpal tunnel syndrome. However, any evidence of axonal loss is classified as severe carpal tunnel syndrome. [14]. Electromyography shows fibrillation and positive sharp spikes in severe compression with muscle atrophy. Nerve conduction may reveal an increase in terminal sensory latency, sensory conduction velocity or motor conduction velocity when compared with the other hand.

No imaging studies are considered routine in the diagnosis of carpal tunnel syndrome. Magnetic resonance imaging of the carpal tunnel is particularly useful preoperatively if a space-occupying lesion in the carpal tunnel is suggested. MRI does not rule out the multitude of other differential diagnoses and it is time consuming and resource intensive. [15] The same thing occurs with the use of ultrasound in the diagnosis of this entity, because there can be 
problems differentiating the median nerve from surrounding soft tissue, and some studies report that it does not correlate well with both clinical and electrodiagnostic criteria, limiting its role in diagnosis. [16]

CTS-6. Diagnostic Clinical Criteria for Carpal Tunnel Syndrome

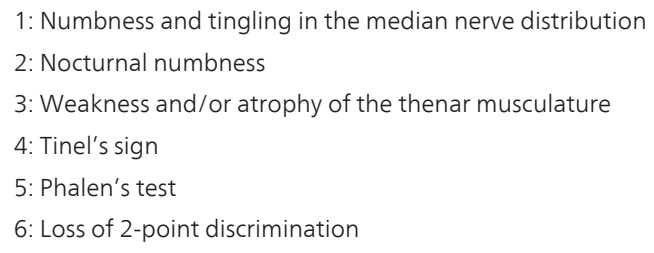

Table 1. Diagnostic Clinical Criteria for Carpal Tunnel Syndrome

\subsubsection{Treatment}

It can be divided in non-operative and surgical decompression of the carpal tunnel. The nonoperative treatment is based in splintage of the wrist in a neutral position for three weeks and steroid injections. This therapy has variable results, with a success rate up to $76 \%$ during one year, but with a recurrence rate as high as $94 \%$. Non-operative treatment is indicated in patients with intermittent symptoms, initial stages and during pregnancy [17].

The only definitive treatment for carpal tunnel syndrome is surgical expansion of the carpal tunnel by transection of the transverse carpal ligament. There is much controversy over what is the most appropriate surgical technique for decompression of the carpal tunnel, either by and open or by an endoscopic approach. In an attempt to resolve this issue, numerous prospective randomized trials have been reported comparing both techniques in terms of safety, efficacy, perioperative morbidity, relative costs and the return to preoperative functional status with variable results. One of the latest studies regarding this matter, was a systematic review performed in 2007 by Sholten et al, published by the Cochrane Collaboration that compared both techniques, reporting equal outcome scores by three months and with rates of complications similar in most studies, concluding there is no strong evidence to support the need for conversion from open techniques to endoscopic or more limited techniques. In addition, some other authors like Atroshi and Trumble have similar conclusions, reporting that both techniques appear to be safe and effective methods of treating carpal tunnel syndrome with no clear long-term differences in outcomes measures to support one method as clearly superior to the other. The decision as to which procedure is most appropriate, therefore, remains a matter of choice for surgeons and patients $[18,19]$.

Other approaches like neurolysis of median nerve have been studied. Mackinnon found that it is not beneficial, with recurrence of symptoms because of internal wound healing. It would just be indicated in patients with thenar atrophy, loss of sensation or the presence of a neuroma. [20] Likewise, synovectomy is just indicated in cases of severe thenosynovytis resulting from rheumatoid arthritis, amyloidosis or renal failure. [21] 


\subsubsection{Complications}

Some of the complications reported can be complex regional pain syndrome, scar pain, pillar pain, infection, injury to the palmar cutaneous branch or to the motor branch of the median nerve, vascular or tendon injury, and recurrence reported in $1 \%$ or less of the patients.

\section{Ulnar nerve}

\subsection{Anatomy}

The ulnar nerve contains fibers from C 8 and $\mathrm{T} 1$ and is the largest terminal branch of the medial cord of the brachial plexus. The nerve enters the arm with the axillary artery and courses medially to the brachial artery before piercing the intermuscular septum approaching the elbow. It then travels along the border of the medial head of the triceps and enters the postcondylar groove lateral to the medial epicondyle [22]. At the elbow, the ulnar nerve enters the forearm between the medial epicondyle and the olecranon through the cubital tunnel. The roof of the cubital tunnel is a fibrous aponeurosis that thickens to form the cubital tunnel retinaculum or arcuate ligament of Osborne. This retinaculum connects the tendinous origin of the humeral and ulnar heads of the flexor carpi ulnaris, giving off branches to the elbow joint [23] (Figure 4).

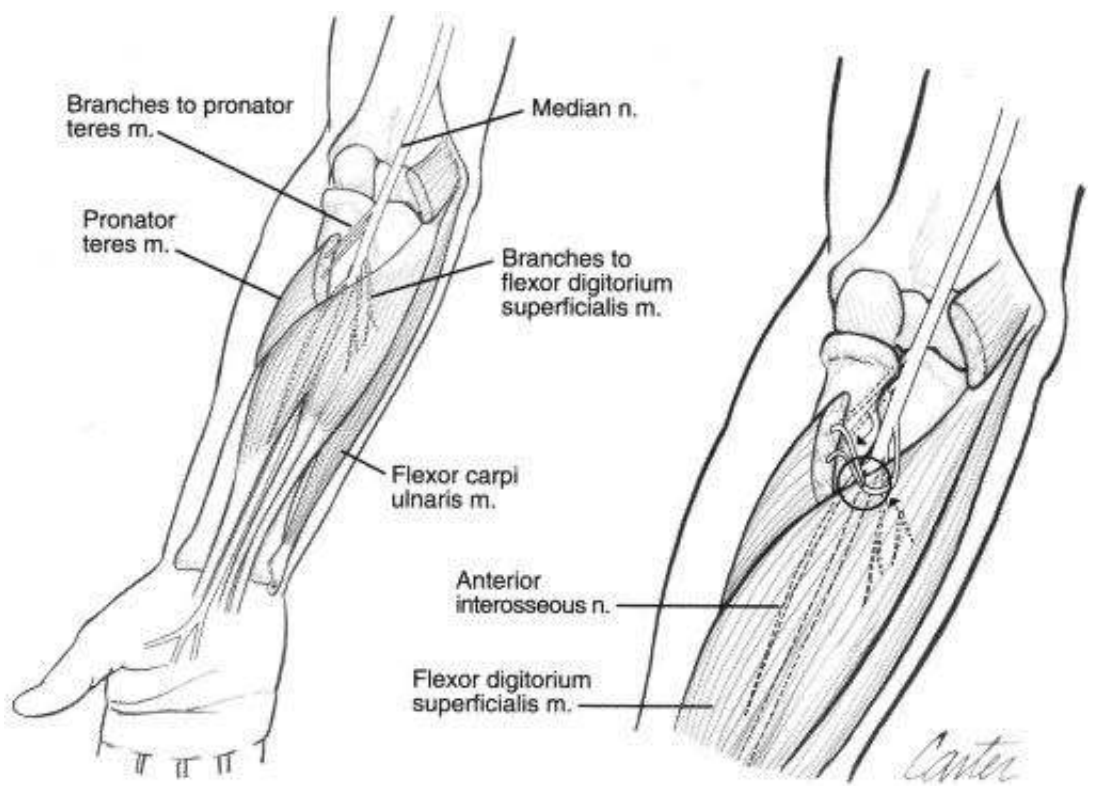

Figure 4. Ulnar nerve anatomy at the elbow. 
Exiting the tunnel, the ulnar nerve pierces the flexor pronator aponeurosis, innervating the flexor digitorum muscles before entering Guyon's canal at the wrist. The terminal branches of the ulnar nerve supply motor innervation to the adductor pollicis, the flexor pollicis brevis, the hypothenar muscles, the third and fourth lumbricalis, and all of the interosseous muscles. The sensory distribution of the nerve includes the palmar and dorsal medial aspects of the hand, often including half of the ring finger (Figure 5).

\subsection{Ulnar nerve entrapment}

The ulnar nerve, like the median nerve, is susceptible to compression neuropathies at proximal and distal levels. Proximally, the most common site of compression is the cubital tunnel as the ulnar nerve enters the forearm between the medial epicondyle and the olecranon. Other potential sites of compression at the elbow, are between the humeral and ulnar heads of the flexor carpi ulnaris muscle and $3 \mathrm{~cm}$ distal to the cubital tunnel, when the ulnar nerve pierces the flexor pronator aponeurosis. Distally, the ulnar nerve can be compressed at the Guyon's canal at the wrist. Each one of these sites of compression present with different signs and symptoms which will be described next.

\subsection{Cubital tunnel syndrome (Ulnar nerve compression at the elbow)}

\subsubsection{Etiology}

The majority of cases occur spontaneously with no documented history of trauma, caused by adhesions that prevent the nerve's gliding with elbow flexion, stretching the nerve behind the epicondyle that impairs nerve conduction. Other causes include direct pressure either by tumors, external swelling-synovium, lipomas or osteophytes, subluxation over the medial epicondyle or just by inadequate space in the cubital tunnel and over the potential sites of compression mentioned above [24].

\subsubsection{Diagnosis}

The patient may present both motor and sensory disturbances, including pain at the medial portion of the proximal third of the forearm, parestesias or anesthesia of palmar and dorsal surfaces of the ring and small fingers, and ulnar innervated intrinsic muscles weakness, which can present atrophy in late stages. During physical exam, the acute flexion of the elbow for 30 seconds usually accentuates the sensory symptoms and also may cause tingling in the little and ring finger, promptly relieved by extending the elbow. A positive Tinel's sign at the posterior elbow will be referred to the small finger.

We may also find a positive Froment's sign and a positive Wartenburg's sign (Figure 6). Froment's sign tests for the action of adductor pollicis, which is weak with an ulnar nerve compression. A patient is asked to hold an object, usually a flat object such as a piece of paper, between their thumb and index finger. The examines then attempts to pull the object out of the patient's hands. A normal individual will be able to maintain a hold on the object without difficulty. With ulnar nerve palsy, the patient will experience difficulty maintaining a hold and 
will compensate by flexing the flexor pollicis longus of the thumb to maintain grip pressure. Clinically, this compensation manifests as flexion of the interphalangeal joint of the thumb.

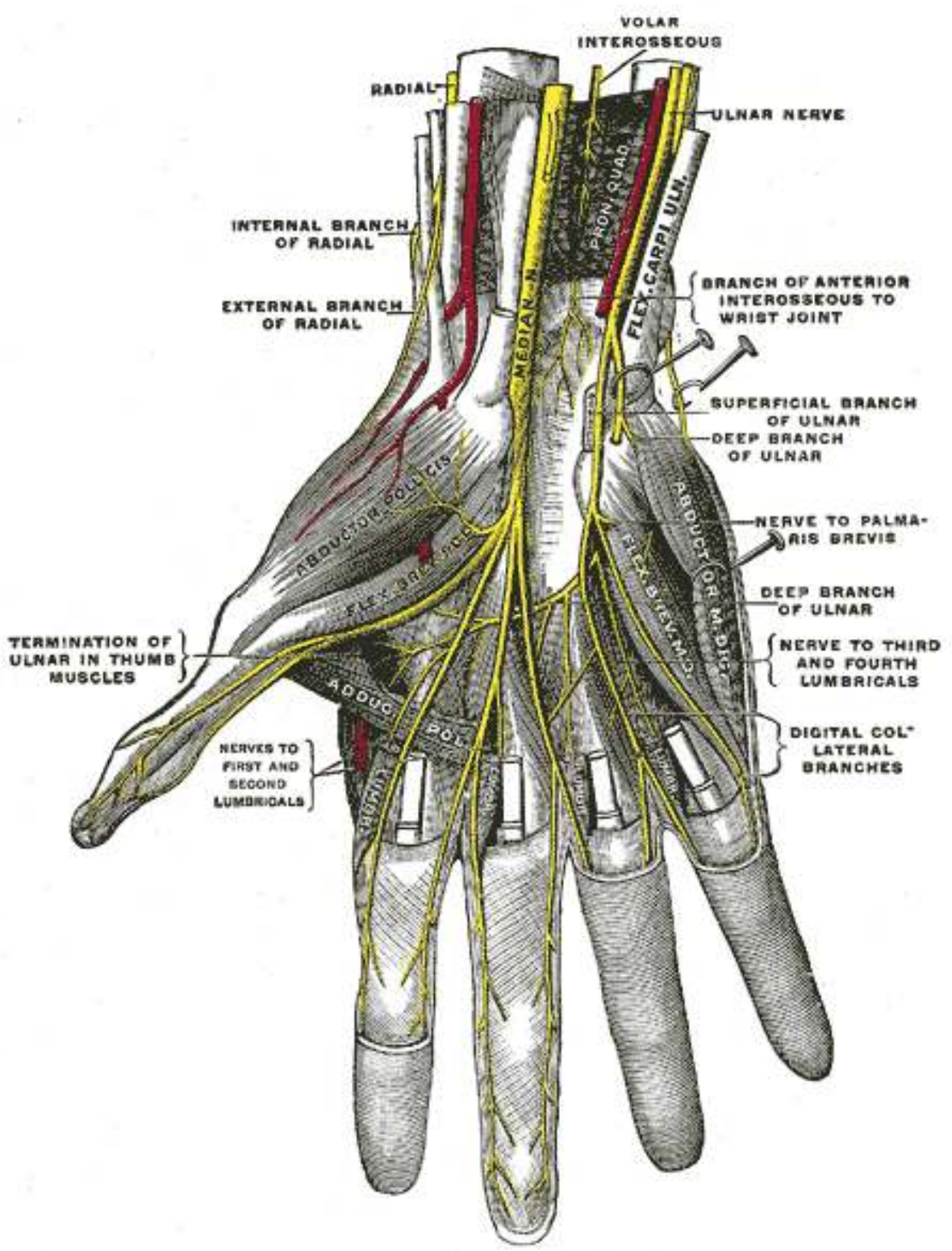

Figure 5. Ulnar nerve anatomy in the hand. 
Simultaneous hyperextension of the thumb metacarpophalangeal joint is indicative of ulnar nerve compromise.

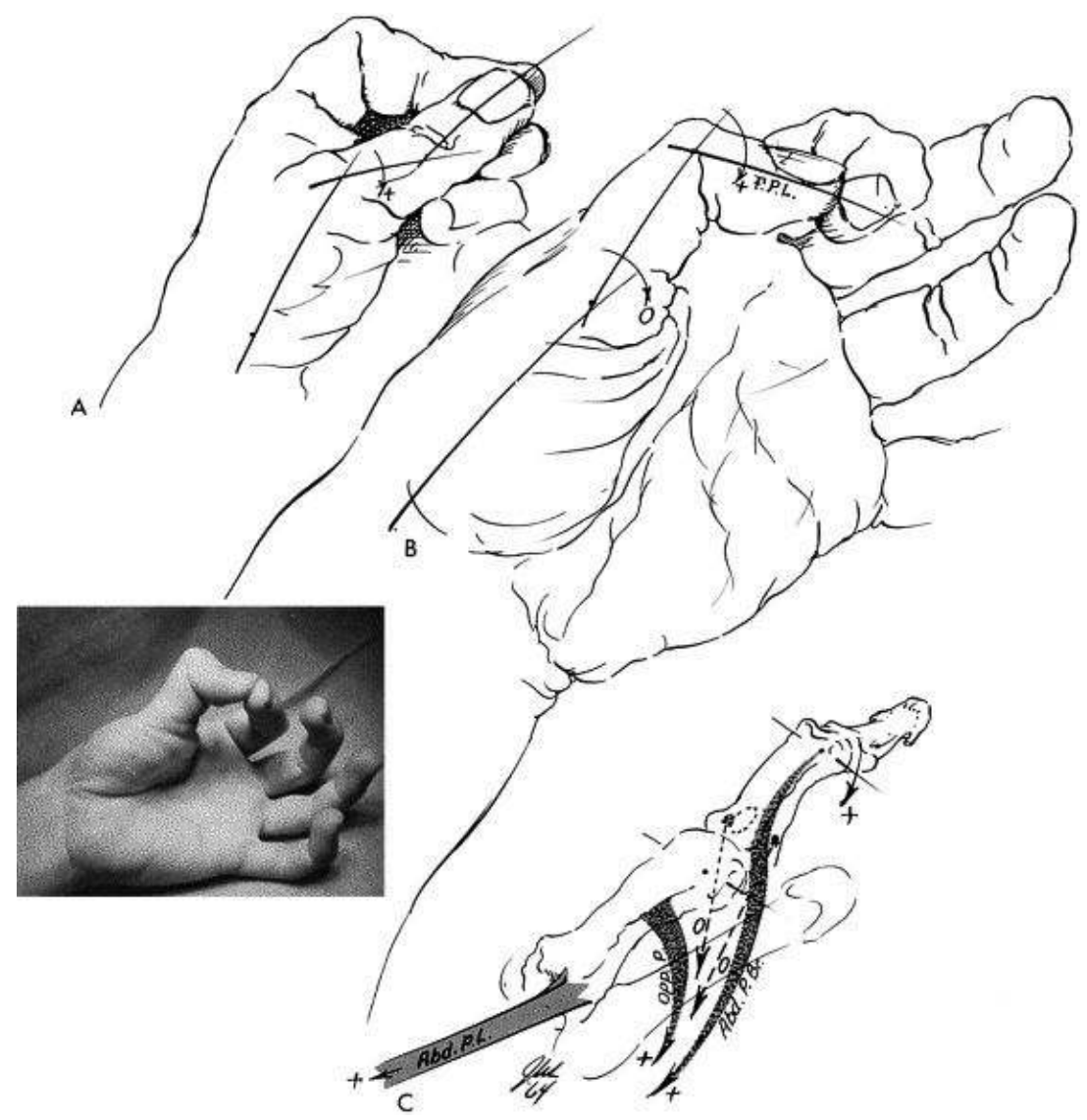

Figure 6. Positive Froment's sign.

On the other hand we have Wartenburg's sign. The patient is placed with wrist in neutral position and forearm fully pronated and instructed to perform full extension of all the fingers. Once digits are extended, patient is asked to fully abduct all fingers and then adduct all fingers. A positive signs is indicated with the observation of abduction of the $5^{\text {th }}$ digit, with inability to adduct the $5^{\text {th }}$ finger when extended. The inability to perform adducted digital extension is due to weakness in ulnar innervated intrinsic muscles.

Electromyography and nerve conduction may reveal a drop in speed conduction or alterations in the sensitive latency, but these studies may be normal, specially in postural conditions, requiring complementary studies like X-rays or an MRI if a space occupying lesion is suspected or if there is a conduction block with established compression but the site is not clear. 


\subsubsection{Treatment}

It is divided in non-operative and operative options. The non-operative treatment is advised in patients with mainly postural symptoms by avoiding flexing the elbow or leaning on the inner side of the elbow, and by splinting the elbow at $45^{\circ}$ extension at night, changing the patient's sleeping posture. One may consider surgery in more advanced stages, if the patient refers numbness or weakness in the hand, which may represent axonal demyelination and muscle atrophy.

Surgical management of the ulnar nerve entrapment at the elbow is determined by the patient's preoperative symptoms and intraoperative findings. It includes transposition of the nerve anterior to the axis of rotation of the elbow so that elbow flexion relaxes rather than stretches the nerve. Commonly performed procedures include simple decompression by unroofing the cubital tunnel, anterior subcutaneous transposition, intramuscular transposition, submuscular transposition, and medial epicondylectomy.

In selected cases, simple decompression of the cubital tunnel and the anterior subcutaneous transposition may be effective, but the ulnar nerve may be more susceptible to trauma injuries as it becomes more superficial. The submuscular anterior transposition is the best operation for cubital tunnel syndrome when an adequate distal mobilization is performed. Other options include a percutaneous and endoscopic release being both technically possible but not generally recommended because of poor results and a high incidence of recurrence [25].

\subsubsection{Complications}

Complications are rare but they include haematoma, infection, neuroma, damage to medial cutaneous nerve of forearm and devascularization of the ulnar nerve, which is the worst of the complications.

\subsection{Ulnar nerve compression at the wrist}

\subsubsection{Guyon's canal}

At the wrist, the ulnar nerve and artery enter Guyon's canal, which is a fibro-osseous tunnel formed between the pisiform and hamate hook. The floor of the canal is formed by the pisohamate ligamento and the flexor retinaculum, and the roof is the palmaris brevis and the superficial volar carpal ligament (continuation of distal forearm fascia).

Within Guyon's canal, the ulnar nerve bifurcates into superficial and deep branches giving off sensory and motor branches, which innervate intrinsic muscles of the hand previously described in this chapter (Figure 7).

\subsubsection{Zones of nerve}

As the ulnar nerve enters the wrist through Guyon's canal, it is divided in 3 zones:

i. $\quad$ Proximal to bifurcation of nerve into deep and superficial branches. 
ii. Around deep motor branch.

iii. Around superficial sensory branch.

\subsubsection{Etiology}

The most common cause of Guyon's canal entrapment is a carpal ganglion. The next most common etiology is repeated trauma to the hypothenar area usually related to occupation. Finally, other less frequent causes include osteophytes from pisotriquetral joint, fracture of the hook of hamate, and pseudoaneurysms of the ulnar artery.

\subsubsection{Diagnosis}

The patient will present with some similar symptoms as in the cubital tunnel syndrome, with some specific differences. In low ulnar neuropathy, the symptoms will not be related to position of the elbow. Also, the sensation at the dorsal aspect of the ulnar border will be preserved, as the dorsal sensory branch of the ulnar nerve has taken off 5 to $10 \mathrm{~cm}$ proximal to Guyon's canal. The function of flexor carpi ulnaris and flexor digitorium profundus muscles will be preserved. The motor affection will be exclusive of the intrinsic muscles of the hand, which can be measured with lateral pinch between thumb and side of index finger.

The diagnosis is mainly clinic but some other studies may be needed in order to complete our investigation. $X$-rays are necessary to evaluate the integrity of the osseous components of the canal, ultrasound if we suspect of a ganglion, arteriogram if ulnar artery aneurysm is suspect-

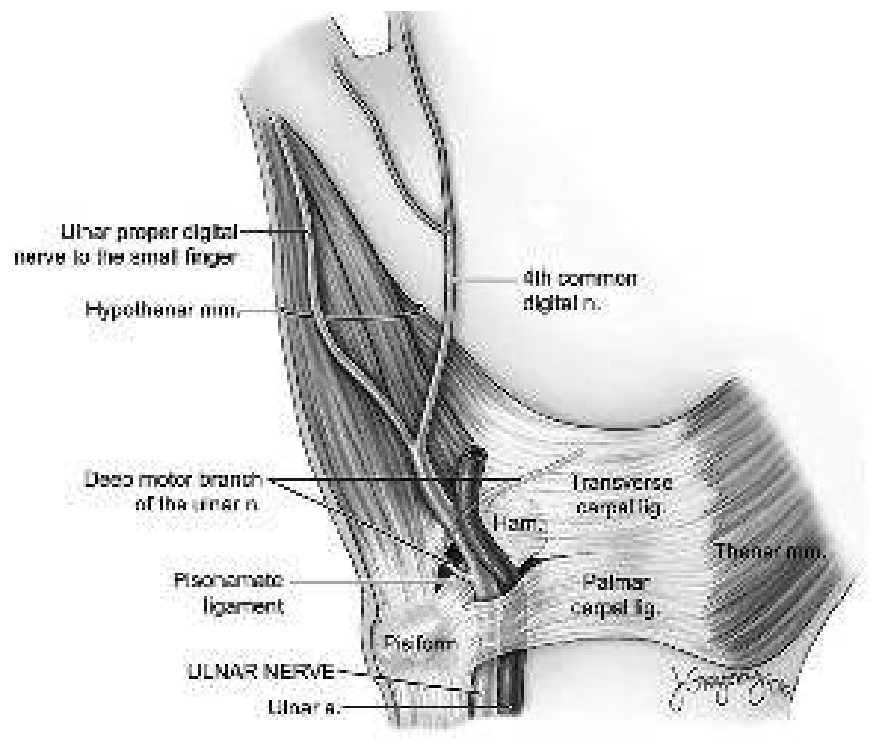

Figure 7. Guyon's canal anatomy. 
ed, MRI if precise location of tumours needs to be addressed, and finally electromyography and nerve conduction to confirm the level of conduction block.

\subsubsection{Treatment}

It consists in surgical decompression of the canal with special care to avoid injury to the dorsal division, which does not pass through the canal. The safest way to decompress the canal is finding the nerve proximal to pisiform and tracing the branches of the nerve distally, progressively unroofing the canal. Once it is open we must treat any pathology we identify like a ganglion or a pseudoanerysm.

\section{Radial nerve}

\subsection{Anatomy}

The radial nerve receives innervation form $\mathrm{C} 5-\mathrm{C} 8$ and $\mathrm{T} 1$ roots, being the terminal branch of the posterior cord. It enters the arm behind the brachial artery, medial to the humerus and anterior to the long head of the triceps muscle running through the radial groove at the humerus, giving off branchesforboth head sof thetricepsmuscle. It descends distally along theborder of thebrachialis muscle and approximately $2 \mathrm{~cm}$ distal to the elbow, the radial nerve divides into the posterior interosseous nerve and the superficial sensory divisions. The posteriorinterosseousnerve passes beneath the fibrous proximal margin of the supinator muscle, known as the arcade of Frohse, and bifurcates to innervate the extensor carpi ulnaris muscle and the digital extensor muscles. The radial nerve does not innervate any hand muscle [26] (Figure 8).

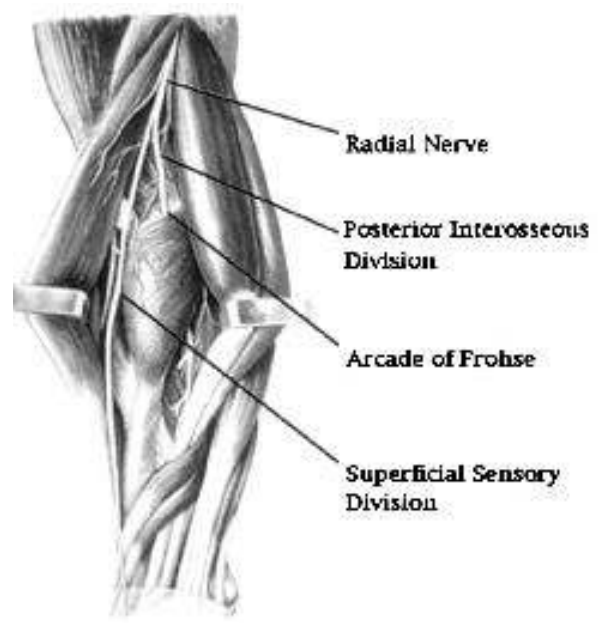

Figure 8. Radial nerve anatomy showing its divisions at the forearm. 


\subsection{Radial nerve entrapment}

Lister et al, in 1979, suggested 4 possible sites of radial nerve compression: the fibrous bands anterior to the radial head, the "radial recurrent fan" of vessels described by Henry, the tendinous margin of the extensor carpi radialis brevis, and the arcade of Frohse. A fifth site of possible compression of this nerve is at the radial tunnel, which represents the fascia at the superficial portion of the supinator muscle that may compress the deep branch of the radial nerve. Nevertheless, the compression of the posterior interosseous branch is the most important entity in this matter (Figure 9).

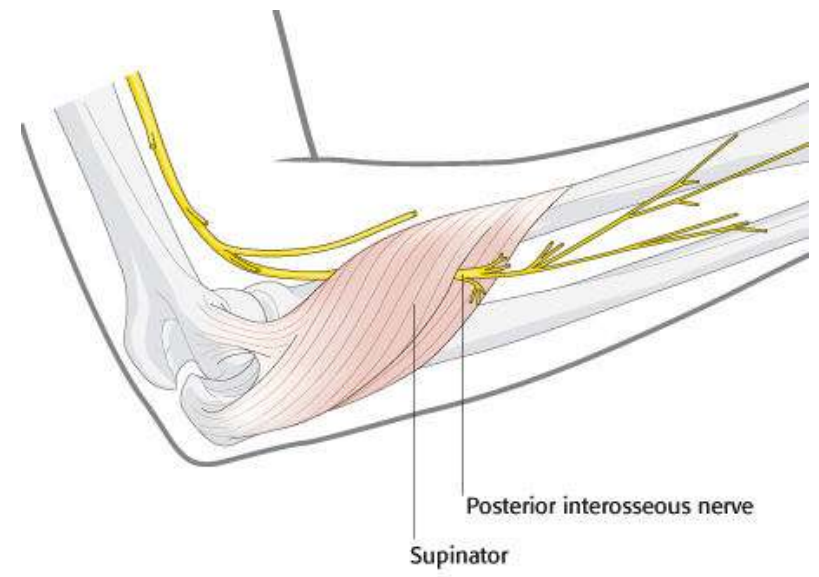

Figure 9. Posterior interosseous branch and its relation with the supinator muscle.

\subsection{Proximal radial nerve compression}

\subsubsection{Etiology}

There are many possible causes of proximal radial nerve compression, being the most common by direct pressure in the axilla, traumatic division, iatrogenic injury or by traction. At the elbow, it may be caused by a fibrous band from the shaft of the humerus that crosses the nerve to the lateral epicondyle [27].

\subsubsection{Diagnosis}

The patient will present with slight weakness of elbow flexion, marked weakness of elbow and wrist extension, finger elevation, thumb retroposition and numbness over the dorsal aspect of thumb base. If the compression is at the elbow, the patient will not have disturbance of the radial wrist extensor muscles as their motor nerves separate from the radial nerve proximal to the elbow, but the sensory branch will be affected as the motor division to the digital extensor muscles. Electrophysiologic studies are not diagnostic unless there is significant denervation. 


\subsubsection{Treatment}

In case of pressure palsy, observation is indicated as most of the symptoms may recover in hours, several weeks or even months. If the patient only presents with moderate symptoms limited to the sensory division of the nerve, a trial of systemic steroids and rest of the arm usually is considered. In severe and progressive cases a surgical decompression may be indicated with a dorsoradial surgical approach [28].

\subsection{Posterior interosseous syndrome}

\subsubsection{Etiology}

Brachial neuritis, fibrous bands anterior to the radial head, fibrous proximal edge of extensor carpi radialis brevis, arcade of Frohse, distal edge of supinator, lipomas and synovitis from proximal radioulnar joint or radioocapitellar joint.

\subsubsection{Diagnosis}

The patient will present with weakness of the hand and wrist often with rapid onset. The wrist extension is preserved but it will move radialwards because of failure of extensor carpi radialis brevis and extensor carpi ulnaris. There will be no elevation of the metacarpophalangeal joints with no retroposition of the thumb. The majority of cases have no sensory disturbance in the distribution of the superficial branch of the radial nerve. Electrophysiologyc studies are of little help; the diagnosis is basically from careful and serial evaluations.

\subsubsection{Treatment}

The management can be divided in operative and non-operative options. Observation is initially indicated if no space-occupying lesion is suspected up to 12 months. It is accompanied by splinting of the wrist in extension or by the use of a dynamic extension splint. Severe or progressive cases need surgical decompression, which has little risk, very low morbidity, and is typically followed by prompt relief from the pain. The surgery consists in a total external neurolysis of the nerve, starting $2 \mathrm{~cm}$ distal to the elbow crease carried through the subcutaneous tissues distally.

\subsection{Wartenburg's syndrome}

\subsubsection{Etiology}

This syndrome originates from compression of superficial radial nerve as it emerges from beneath brachioradialis muscle to reach the subcutaneous plane over the radial border of the distal forearm. At the point of exit from beneath the muscle, a compression of the nerve can develop. It does not develop spontaneously, but is an infrequent complication of trauma to the midforearm. 


\subsubsection{Diagnosis}

The patient will present local pain and sensory disturbance to the dorsal-lateral skin of the hand, with tingling over back of thumb base, with a positive Tinel's sign at the point of exit of the nerve from beneath the braquiradialis muscle. As this muscle is a supinator muscle pain is accentuated by attempting this motion while the forearm is passively pronated. Electrophisiologic tests reveal reduced conductions and are generally not necessary for diagnosis.

\subsubsection{Treatment}

Surgical decompression using a dorsoradial approach. The superficial radial nerve is identified and released at it emerges beneath the brachioradialis tendon. The prognosis is excellent.

\section{Conclusion}

Compression neuropathies are one of the most prevalent disorders of the peripheral nervous system with an increasing incidence over the past decades. Recent studies have helped clarify the diagnosis and treatment for many of these neuropathies, facilitating a prompt recognition of the signs and symptoms, achieving an accurate diagnosis and a prompt treatment before the establishment of complications. The ability to recognize nerve entrapment syndromes and to distinguish them from other diseases of peripheral nerves, are important clinical skills. Although electrophysiological assessments are important in the diagnosis of neuropathies, our clinical skills remain the most reliable tool to identifying them and start an accurate treatment protocol. It is always important to begin with a non-operative strategy in patients with mild symptoms, nevertheless, surgical decompression is the definitive treatment of choice for most of the compression neuropathies, that is why it is important to know all the surgical alternatives and know the surgical anatomy for each upper extremity nerve.

\section{Author details}

Javier López Mendoza and Alexandro Aguilera Salgado

Postgraduate Course in Plastic and Reconstructive Surgery, Universidad Nacional Autónoma de México, Hospital General “Dr. Manuel Gea González“, México City, Mexico

\section{References}

[1] David Warwick, MD. Compression Neuropathy. In: Oxford University Press. Oxford Specialist Handbooks in Surgery. Hand Surgery. (2009). , 314-331. 
[2] Charles, H, \& Thorne, M. D. Compression Neuropathies in the Upper Limb and Electrophisiologic Studies. In: Lippincott Williams \& Wilkins. Grabb and Smith's Plastic Surgery. Sixth Edition. (2007). , 849-853.

[3] Robert, J, \& Spinner, M. D. Compressive neuropathies of the upper extremity. Clin Plastic Surg (2003). , 30(2003), 155-173.

[4] David Green MD. Compression Neuropathies. In: Churchill Livingstone. El Sevier. Green's Operative Hand Surgery. (2005). Fifth Edition. , 999-1045.

[5] Karol, A, \& Gutowski, M. D. Hand II: Peripheral Nerves and Tendon Transfers. In: Selected Readings in Plastic Surgery. (2003). , 9(33), 19-32.

[6] Brian McNamara, MD. Clinical Anatomy Of The Median Nerve. ACNR (2003). http://www.acnr.co.uk/pdfs/volume2pdfaccessed 19 August 2012).

[7] Akira NaganoSpontaneous Anterior Interroseous Nerve Palsy. The Journal Of Bone And Joint Surgery. (2003). B:313-8., 85.

[8] Spinner, M. Injuries to the Major Branches of Peripheral Nerves of the Forearm. Philadelphia: Saunders, (1978). , 160-227.

[9] Douglas, H. C. L. Anterior Interosseous Nerve Syndrome. Journal of The American Society For Surgery of the Hand. November (2001). , 1(4)

[10] American Academy of Orthopaedic Surgeons Work Group PanelClinical guidelines on diagnosis of carpal tunnel syndrome. Available at: www.aaos.org/research/guidelines/CTS_guideline.pdf.Accessed November 27, (2012).

[11] Jaimie, T, \& Shores, M. D. An Evidence-Based Approach to Carpal Tunnel Syndrome. Plast. Reconstr. Surg. (2010). , 126, 2196-2204.

[12] Massy-westropp, N, Grimmer, K, \& Bain, G. A systematic review of the clinical diagnostic tests for carpal tunnel syndrome. J Hand Surg (2000). A:, 120-127.

[13] Kyle, D, \& Bickel, M. D. Carpal Tunnel Syndrome. J Hand Surg (2010). A:, 147-152.

[14] Robinson, L. R. Electrodiagnosis of Carpal Tunnel Syndrome. Phys Med Rehabil Clin N Am. Nov (2007).

[15] Zagnoli, F, \& Andre, V. Le Dreff P, et al. Idiopathic Carpal Tunnel Syndrome. Clinical, electrodiagnostic, and magnetic resonance imaging correlations. Rev Rhum Engl Ed. Apr (1999). , 66(4), 192-200.

[16] Lee, D, Van Holsbeeck, M. T, Janevski, P. K, et al. Diagnosis of Carpal Tunnel Syndrome. Ultrasound Versus Electromyography. Radiol Clin North Am. Jul (1999). , 37(4), 859-72.

[17] Scholten, R. J. Mink van der Molen A, Uitdehaag BM, Bouter LM, de Vet HC. Surgical treatment options for carpal tunnel syndrome. Cochrane Database Syst Rev (2007). CD003905. 
[18] Trumble, T. E, Diao, E, Abrams, R. A, \& Gilbert-anderson, M. M. Single-portal endoscopic carpal tunnel release compared with open release: A prospective, randomized trial. J Bone Joint Surg Am. (2002). A:1107-1115., 84.

[19] Atroshi, I, Larsson, G. U, Ornstein, E, Hofer, M, Johnsson, R, \& Ranstam, J. Outcomes of endoscopic surgery compared with open surgery for carpal tunnel syndrome among employed patients: Randomised controlled trial. BMJ. (2006).

[20] Mackinnon, S. E, Mccabe, S, Murray, J. F, et al. Internal neurolysis fails to improve the results of primary carpal tunnel decompression. J Hand Surg Am 16:211-218, (1991).

[21] Charlotte Shum, MD et al. The Role of Flexor Tenosynovectomy in the Operative Treatment of Carpal Tunnel Syndrome. The Journal of Bone and Joint Surgery (American) 84:221-225 ((2002).

[22] Daniel, B, \& Polatsch, M. D. Ulnar Nerve Anatomy. Hand Clin (2007). , 23(2007), 283-289.

[23] Gonzalez, M. H, Lotfi, P, Bendre, A, \& Mandelbroyt, Y. Lieska N: The ulnar nerve at the elbow and its local branching: An anatomic study. J Hand Surg [Br] 26B:(2001). , 142-144.

[24] Jason, H, \& Huang, M. D. Ulnar Nerve Entrapment Neuropathy At The Elbow: Simple Decompression. Neurosurgery. (2004). , 55, 1150-1153.

[25] Lowe JB III, Maggi SP, Mackinnon SE. The position of crossing branches of the medial antebrachial cutaneous nerve during cubital tunnel surgery in humans. Plast Reconstr Surg (2004). , 114(3), 692-6.

[26] Keith, L. Moore. Arm, Forearm and Hand. In: Lippincott Williams \& Wilkins. Anatomy With Clinical Orientation. (2004). Fourth Edition. , 730-796.

[27] Brian Rinker, MD. Proximal Radial Compression Neuropathy. Ann Plast Surg (2004). , 52, 174-180.

[28] Lister, G. D, Belsole, R. B, \& Kleinert, H. E. The radial tunnel syndrome. J Hand Surg. (1979). , 4, 52-59. 